\title{
The Missing Piece Syndrome in Peer-to-Peer Communication
}

\author{
Bruce Hajek and Ji Zhu \\ Department of Electrical and Computer Engineering \\ and the Coordinated Science Laboratory \\ University of Illinois at Urbana-Champaign
}

\begin{abstract}
Typical protocols for peer-to-peer file sharing over the Internet divide files to be shared into pieces. New peers strive to obtain a complete collection of pieces from other peers and from a seed. In this paper we investigate a problem that can occur if the seeding rate is not large enough. The problem is that, even if the statistics of the system are symmetric in the pieces, there can be symmetry breaking, with one piece becoming very rare. If peers depart after obtaining a complete collection, they can tend to leave before helping other peers receive the rare piece. Assuming that peers arrive with no pieces, there is a single seed, random peer contacts are made, random useful pieces are downloaded, and peers depart upon receiving the complete file, the system is stable if the seeding rate (in pieces per time unit) is greater than the arrival rate, and is unstable if the seeding rate is less than the arrival rate. The result persists for any piece selection policy that selects from among useful pieces, such as rarest first, and it persists with the use of network coding.
\end{abstract}

\section{INTRODUCTION}

Peer-to-peer (P2P) communication in the Internet is provided through the sharing of widely distributed resources typically involving end users' computers acting as both clients and servers. In an unstructured peer-to-peer network, such as BitTorrent [2], a file is divided into many pieces. Seeds, which hold all pieces, distribute pieces to peers. New peers continually arrive into the network; they simultaneously download pieces from a seed or other peers and upload pieces to other peers. Peers exit the system after they collect all pieces.

Determining whether a given P2P network is stable can be difficult. Roughly speaking, the aggregate transfer capacity scales up in proportion to the number of peers in the network, but it has to be in the right places. Many P2P systems have performed well in practice, and they incorporate a variety of mechanisms to help achieve stability. A broad problem, which we address in part, is to provide a better understanding of which mechanisms are the most effective under various network settings. These mechanisms include

- Rarest first piece selection policies, such as the one implemented in BitTorrent, whereby peers determine which pieces are rarest among their neighbors and preferentially download such pieces.

- Tit-for-tat participation constraints, such as the one implemented in BitTorrent, whereby peers are choked off from receiving pieces from other peers unless they upload pieces to those same peers. This mechanism provides an important incentive for peers to participate in uploading pieces, but it may also be beneficial in balancing the distribution of pieces.

- Peers dwelling in the network after completing download, to provide extra upload capacity.

This work was supported in part by the National Science Foundation under grant NSF ECS 06-21416 and CCF 10-16959. 
- Network coding [1], [4], whereby data pieces are combined to form coded pieces, giving peers numerous ways to collect enough information to recover the original data file.

This paper determines what parameter values yield stability for a simple model of a P2P file sharing network. The main model does not include the enhancements mentioned in the previous paragraph, but extensions and discussion regarding the above mechanisms is given. The model includes a fixed seed in the network that uploads with a constant rate. New peers arrive according to a Poisson process, and have no pieces at the time of arrival. Random peer contact is assumed; each peer contacts a randomly selected target peer periodically. Random useful piece selection is also assumed; each peer chooses which piece to download uniformly at random from the set of pieces that its selected target has and it itself does not have. As in the BitTorrent system, we assume that new peers arrive with no pieces; in effect a peer must first obtain a piece from another peer or the fixed seed before it can begin uploading to other peers. We also assume that peers depart as soon as they have completed their collection.

In a P2P network, the last few pieces to be downloaded by a peer are often rare in the network, so it usually takes the peer a long time to finish downloading. This phenomenon has been referred to as the delay in endgame mode [2] (or last piece problem). We refer to the specific situation that there are many peers in the network and most of them are missing only one piece which is the same for all peers, as the missing piece syndrome. In that situation, peers lucky enough to get the missing piece usually depart immediately after getting the piece, so their ability to spread the missing piece is limited.

The main result in this paper is to show, as suggested by the missing piece syndrome, that the bottleneck for stability is the upload capacity of the seed. Specifically, if the arrival rate of new peers is greater than the seed upload rate, the number of peers in the system converges to infinity almost surely; if the arrival rate of new peers is less than the seed upload rate, the system is positive recurrent and the mean number of peers in the system in equilibrium is finite. The next section gives the precise problem formulation, simulation results illustrating the missing piece syndrome, and the main proposition. The proposition is proved in Sections III and IV] with the help of some lemmas given in the appendix. Section $\mathrm{V}$ provides extensions of the result, including consideration of the enhancement mechanisms mentioned above. In particular, it is shown that the region of network stability is not increased if rarest first piece selection policies, or network coding policies, are applied. Section $\mathrm{V}$ also provides a conjecture regarding a refinement of the main proposition for the borderline case when the arrival rate is equal to the seeding rate; it is suggested that whether the system is stable then depends on the rate that peers contact each other.

The model in this paper is similar to the flat case of the open system of Massoulié and Vojnović [9], [10]. The model in [9], [10] is slightly different in that, rather than having a fixed seed, it assumes that new peers each arrive with a randomly selected piece. A fluid model, based on the theory of density-dependent jump Markov processes (see [7]), is derived and studied in [9], [10]. It is shown that there is a finite resting point of the fluid ordinary differential equation. The analysis in this paper is different and complementary. Rather than appealing to fluid limits, we focus on direct stochastic analysis methods, namely using coupling to prove transience for some parameter values and the Foster-Lyapunov stability criterion to prove positive recurrence for complementary parameter values. Furthermore, our work shows the importance of considering asymmetric sample paths even for symmetric system dynamics. Forthcoming work described in [17] provides analysis of $\mathrm{P} 2 \mathrm{P}$ networks with peers having pieces upon arrival, as in [9], [10], and with peers remaining for some time in the system after obtaining a complete collection.

Some other works related to stability and the missing piece syndrome are the following. The instability phenomenon identified in this paper was discovered independently by Norros et al. 
[13]. Norros et al. [13] proved a version of our main proposition for a similar model, for the case of two pieces. In the model of [13] a peer receives one piece on arrival, with the distribution of the piece number (either one or two) being determined by sampling uniformly from the group consisting of a fixed seed and the population of peers already in the system.

Menasché et al [11] pointed out that in their simulation studies, their "smooth download assumption" and "swarm sustainability" break down if the seed upload rate is not sufficiently large. Leskelä et al. [8] investigate stability conditions for a single piece file, or a two piece file when the pieces are obtained sequentially, when peers remain in the system for some time after obtaining the piece. The earliest papers to analytically study unstructured peer-to-peer files systems with arrivals of new peers are [14], [15]. These papers provide simple models in which a two dimensional differential equation is used that does not take into account the stages of service as peers gain more pieces.

\section{MODEL FORMULATION AND SIMULATIONS}

The model in this paper is a composite of models in [9], [10], [16]. It incorporates Poisson arrivals, fixed seed, random uniform contacts, and random useful piece selection, as follows. The parameters of the model are an integer $K \geq 1$ and strictly positive constants $\lambda, \mu$, and $U_{s}$.

- There are $K$ pieces and $\mathcal{F}=\{1, \ldots, K\}$, so that $\mathcal{F}$ indexes all the pieces.

- The set of proper subsets of $\mathcal{F}$ is denoted by $\mathcal{C}$.

- A peer with set of pieces $c$, for some $c \in \mathcal{C}$, is called a type $c$ peer.

- A type $c$ peer becomes a type $c \cup\{i\}$ peer if it downloads piece $i$ for some $i \notin c$.

- A Markov state is $\mathbf{x}=\left(x_{c}: c \in \mathcal{C}\right)$, with $x_{c}$ denoting the number of type $c$ peers, $|\mathbf{x}|$ denoting the number of peers in the system, and $\mathcal{S}=\mathbb{Z}_{+}^{\mathcal{C}}$ denoting the state space of the system.

- Peers arrive exogenously one at a time with no pieces; the times of arrival form a rate $\lambda$ Poisson process.

- Each peer contacts other peers, chosen uniformly at random from among all peers, for opportunities to download a piece (i.e. pull) from the other peers, according to a Poisson process of rate $\mu>0$. Mathematically, an equivalent assumption is the following. Each peer contacts other peers, chosen uniformly at random from among all peers, for opportunities to upload a piece (i.e. push) to the other peers, according to a Poisson process of rate $\mu>0$.

- Downloads are modeled as being instantaneous. This assumption is reasonable in the context of the previous assumption.

- Random useful piece selection is used, meaning that when a peer of type $c$ has an opportunity to download a piece from a peer of type $s$, the opportunity results in no change of state if $s \subset c$. Otherwise, the type $c$ peer downloads one piece selected at random from $s-c$, with all $|s-c|$ possibilities having equal probability.

- There is one fixed seed, which at each time in a sequence of times forming a Poisson process of rate $U_{s}$, selects a peer at random and uploads a random useful piece to the selected peer.

- Peers leave immediately after obtaining a complete collection.

Given a state $\mathbf{x}$, let $T_{0}(\mathbf{x})$ denote the new state resulting from the arrival of a new peer. Given $c \in \mathcal{C}, 1 \leq i \leq K$ such that $i \notin c$, and a state $\mathbf{x}$ such that $x_{c} \geq 1$, let $T_{c, i}(\mathbf{x})$ denote the new state resulting from a type $c$ peer downloading piece $i$. The positive entries of the generator matrix 
$Q=\left(q\left(\mathbf{x}, \mathbf{x}^{\prime}\right): \mathbf{x}, \mathbf{x}^{\prime} \in \mathcal{S}\right)$ of the Markov process are given by:

$$
\begin{aligned}
q\left(\mathbf{x}, T_{0}(\mathbf{x})\right) & =\lambda \\
q\left(\mathbf{x}, T_{c, i}(\mathbf{x})\right) & =\frac{x_{c}}{|\mathbf{x}|}\left(\frac{U_{s}}{K-|c|}+\mu \sum_{s: i \in s} \frac{x_{s}}{|s-c|}\right) \\
& \text { if } x_{c}>0 \text { and } i \notin c .
\end{aligned}
$$

To provide some intuition, we present some simulation results. Figure 1 shows simulations of the system for $U_{s}=\mu=1$ and $K=40$ pieces. The first plot shows apparently stable behavior.
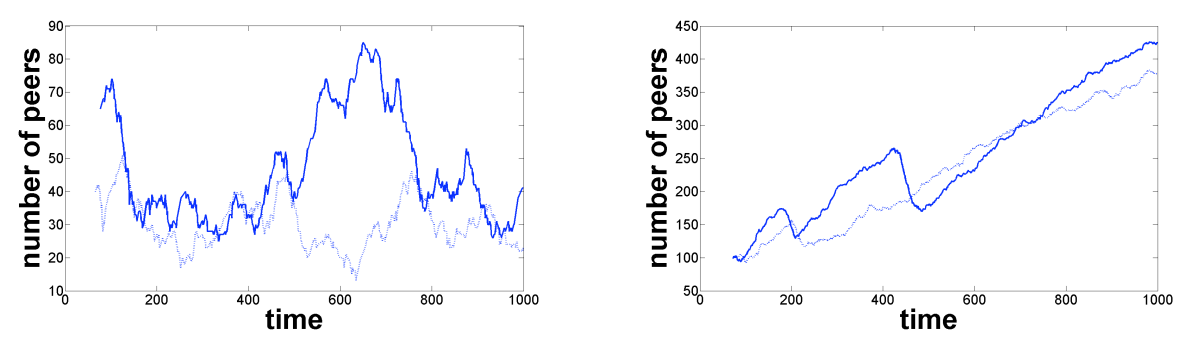

Fig. 1. Number of peers vs. time. The first plot is for $\lambda=0.6$ (dashed) and $\lambda=0.8$ (solid), and the second is for $\lambda=1.2$ (dashed) and $\lambda=1.4$ (solid).

After an initial spike, the number of peers in the system seems to hover around 30 (for $\lambda=0.6$ ) or 45 (for $\lambda=0.8$ ), which by Little's law is consistent with a mean time in system around 50 to 60 time units (or about $25 \%$ to $50 \%$ larger than the sum of the download times). However, the second plot shows that for $\lambda=1.2$ or $\lambda=1.4$, the number of peers in the system does not appear to stabilize, but rather to grow linearly. The explanation for this instability is indicated in Figure 2, which shows the time-averaged number of peers that held each given piece during the simulations, for $\lambda=0.6$ in the first plot and for $\lambda=1.4$ in the second plot. The first plot shows that the 40 pieces had nearly equal presence in the peers, with piece 7 being the least represented. The second plot shows that 39 pieces had nearly equal presence and most of the peers had these pieces most of the time, but only a small number of peers held piece 3 . The following proposition, which is the main result of this paper, confirms that the intuition behind the simulation results is correct.

Proposition II.1 (i) If $\lambda>U_{s}$ then the Markov process is transient, and the number of peers in the system converges to infinity with probability one. (ii) If $\lambda<U_{s}$ the Markov process with generator $Q$ is positive recurrent, and the equilibrium distribution $\pi$ is such that $\sum_{\mathbf{x}} \pi(\mathbf{x})|\mathbf{x}|<\infty$.

In the remainder of this section, we give an intuitive explanation for the proposition, which also guides the proof. We first give an intuitive justification of Proposition II.1 (i), so assume $\lambda>U_{s}$. Under this condition, eventually, due to random fluctuations, there will be many peers in the system that are all missing the same piece. While any of the $K$ pieces could be the missing one, to be definite we focus on the case that the peers are missing piece one. A peer is said to be in the one $c l u b$, or to be a one-club peer, if it has all pieces except piece one. We consider the system starting from an initial state in which there are many peers in the system, and all of them are in the one club. The system then evolves as shown in Figure 3. The large size of the box showing the one club indicates that most peers are one club peers. A peer not in the one club is said to be a young peer, and a young peer is said to be normal if it does not have piece one and infected if 

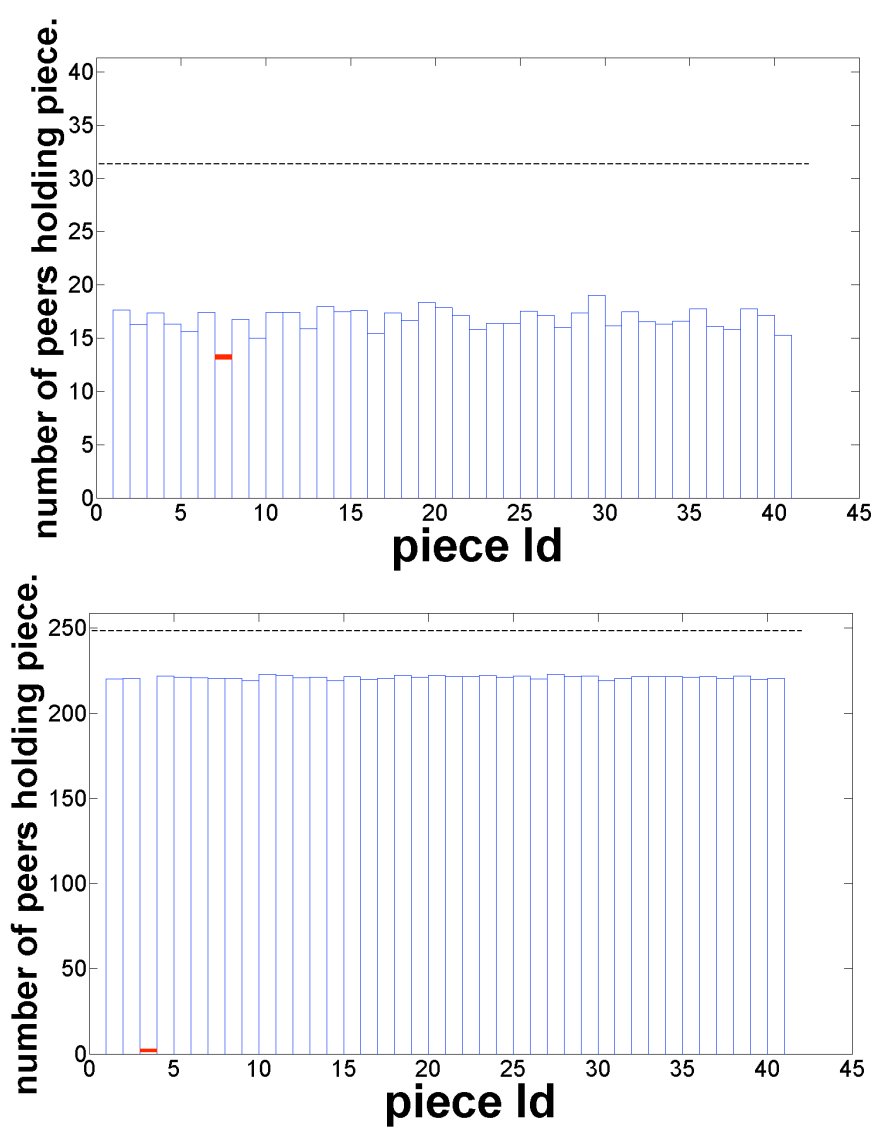

Fig. 2. Average number of peers holding each piece for the duration of the simulations. The first plot is for $\lambda=0.6$ and the second is for $\lambda=1.4$. The dashed lines indicate time-average number of peers in system.

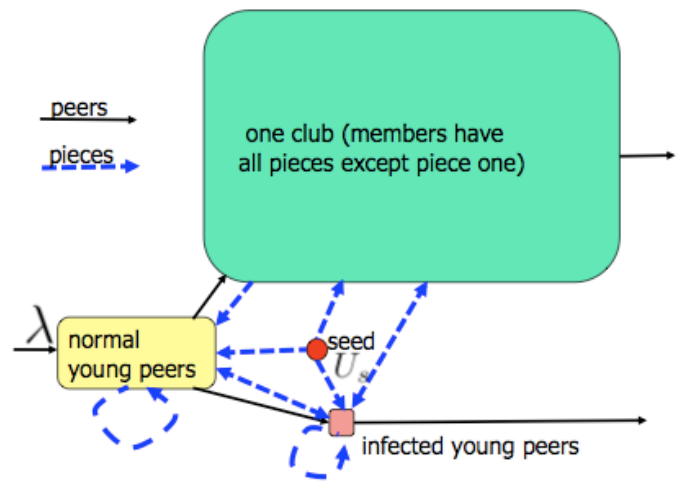

Fig. 3. Flows of peers and pieces. Solid lines indicate flows of peers; dashed lines indicate flows of pieces.

it does have piece one. Since there are so many one club peers to download from, a peer doesn't stay young very long; most of the young peers join the one club soon after arrival. However, due to the fixed seed uploading pieces, some of the normal young peers become infected peers. Those infected peers can infect yet more young peers, thereby forming a branching process. But typically the infected young peers do not infect other young peers, so that the branching process is highly subcritical. Therefore, the rate of departures from the one club due to uploads of piece 
one from infected peers is small. Therefore, most peers eventually enter the one club, and the main way that peers leave the one club is to receive piece one directly from the fixed seed. So the long term arrival rate at the one club is close to $\lambda$ and the departure rate from the one club is close to $U_{s}$. Therefore, the one club can grow at rate close to $\lambda-U_{s}$, while the number of young peers will stay about constant. These ideas are made precise in the proof.

To understand why the system is stable for $\lambda<U_{s}$, the rough idea is to show that whenever there are many peers in the system, no matter what the distribution of pieces they hold, the system moves towards emptying out. If there are many peers in the system, one of the following two cases holds. The first case is that most of the peers have the same number, say $k_{o}$, of pieces. Intuitively, the worst case would be for all peers with $k_{o}$ pieces to have identical collections of pieces, in which case no peer with $k_{o}$ pieces would be useful to another. However, if $\lambda<U_{s}$, such a state can't persist, because peers with $k_{o}$ pieces get additional pieces from the fixed seed at an aggregate rate near $U_{s}$, while the long term rate that new peers with exactly $k_{o}$ pieces can appear is less than or equal to $\lambda$. If the system is not in the first case just described, then there are at least two sizeable groups of peers, so that all the peers in the first group have one number of pieces and all peers in the second group have some larger number of pieces. Then all peers in the second group can be helpful to any peer in the first group, so that there will be a large rate of downloads. Thus, if there are many peers in the system, no distribution of the pieces they hold can persist. To prove stability, it is still necessary to show that the state can't spiral out to ever increasing loads through some quasi-periodic behavior. This is achieved through the use of a potential function and the Foster-Lyapunov stability criterion.

\section{PROOF OF INSTABILITY IF $\lambda>U_{s}$}

Proposition II.1(i) is proved in this section; it can be read independently of the proof of Proposition II.1(ii) in the next section. The proof follows along the lines of the intuitive explanation given just after the statement of the proposition in Section II and an additional explanation of the proof is provided in a remark at the end of the section. Assume $\lambda>U_{s}$. If $K=1$, the system reduces to an $M / M / 1$ queueing system with arrival rate $\lambda$ and departure rate $U_{s}$, in which case the number of peers in the system converges to infinity with probability one. So for the remainder of this proof assume $K \geq 2$. To begin:

- Select $\epsilon>0$ so that $3 \epsilon<\lambda-U_{s}$.

- Select $\xi>0$ so that $\epsilon-4 K \xi U_{s}>0$, and

$$
\rho<\frac{1}{2} \text { where } \rho=2 \xi(K-1) .
$$

It follows from (1) that $\xi<0.5$.

- Select $\epsilon_{o}$ small enough that $\frac{\epsilon_{o}}{\lambda-U_{s}-3 \epsilon}<\xi$.

- Select $B$ large enough that

$$
\begin{aligned}
\frac{e^{\lambda[2(K-1) / \mu+1]} 2^{-B}}{1-2^{-\epsilon_{o}}} & \leq 0.1, \\
\frac{64 K^{2} \xi U_{s}}{2 B\left(\epsilon-4 K \xi U_{s}\right)} & \leq 0.1, \\
\frac{\lambda}{2 B \epsilon} \leq 0.1, \text { and } \frac{U_{s}}{2 B \epsilon} & \leq 0.1 .
\end{aligned}
$$

- Select $N_{o}$ large enough that $\frac{B}{N_{o}-3 B} \leq \xi$. 
We shall use the notions of one club, young peer, and infected young peer, as described in the paragraph after Proposition II.1. For a given time $t \geq 0$, define the following random variables:

- $A_{t}$ : cumulative number of arrivals, up to time $t$

- $N_{t}$ : number of peers at time $t$

- $Y_{t}$ : number of young peers at time $t$

- $D_{t}$ : cumulative number of uploads of piece one by infected peers, up to time $t$

- $Z_{t}$ : cumulative number of uploads of piece one by the fixed seed, up to time $t$

The system is modeled by an irreducible, countable-state Markov process. A property of such random processes is that either all states are transient, or no state is transient. Therefore, to prove Proposition II.1(i), it is sufficient to prove that some particular state is transient. With that in mind, we assume that the initial state is the one with $N_{o}$ peers, and all of them are one-club peers. Let $\tau$ be the extended stopping time defined by $\tau=\min \left\{t \geq 0: Y_{t} \geq \xi N_{t}\right\}$, with the usual convention that $\tau=\infty$ if $Y_{t}<\xi N_{t}$ for all $t$. It suffices to prove that

$$
P\left\{\tau=\infty \text { and } \lim _{t \rightarrow \infty} N_{t}=+\infty\right\} \geq 0.6 .
$$

The equation (5) depends on the transition rates of the system out of states such that $Y<\xi N$. Thus, we can and will prove (5) instead for an alternative system, that has the same initial state, and the same out-going transition rates for all states such that $Y<\xi N$, as the original system. The alternative system is defined by modifying the original system by letting the rate of downloads from the set of one-club peers by each young peer be $\mu \max \left\{\frac{N-Y}{N}, \frac{1}{2}\right\}$, and the aggregate rate of downloads from the fixed seed to the set of young peers be $U_{s} \min \left\{\frac{Y}{N}, \xi\right\}$. Note that the rates used for this definition are equal to the original ones on the states such that $Y<\xi N$, as required. The alternative system has the following two properties:

1) Each young peer receives opportunities to download from one-club peers at rate greater than or equal to $\mu / 2$.

2) The fixed seed contacts the entire population of young peers at aggregate rate less than or equal to $\xi U_{s}$.

For the remainder of this proof we consider the alternative system, but for brevity of notation, use the same notation for it as for the original system, and refer to it as the original system.

The following four inequalities will be established, for $\epsilon, \xi, \epsilon_{o}, B$, and $N_{o}$ satisfying the conditions given near the beginning of the section.

$$
\begin{array}{r}
P\left\{A_{t}>-B+(\lambda-\epsilon) t \text { for all } t \geq 0\right\} \geq 0.9 \\
P\left\{Z_{t}<B+\left(U_{s}+\epsilon\right) t \text { for all } t \geq 0\right\} \geq 0.9 \\
P\left\{Y_{t}<B+\epsilon_{o} t \text { for all } t \geq 0\right\} \geq 0.9 \\
P\left\{D_{t}<B+\epsilon t \text { for all } t \geq 0\right\} \geq 0.9
\end{array}
$$

Let $\mathcal{E}$ be the intersection of the four events on the left sides of (6)-(9). Since $N_{t}$ is greater than or equal to the number of peers in the system that don't have piece one, on $\mathcal{E}$, $N_{t} \geq N_{o}+A_{t}-D_{t}-Z_{t}>N_{o}-3 B+\left(\lambda-U_{s}-3 \epsilon\right) t$ for all $t \geq 0$. Therefore, on $\mathcal{E}$, for any $t \geq 0$

$$
\begin{aligned}
\frac{Y_{t}}{N_{t}} & <\frac{B+\epsilon_{o} t}{N_{o}-3 B+\left(\lambda-U_{s}-3 \epsilon\right) t} \\
& \leq \max \left\{\frac{B}{N_{o}-3 B}, \frac{\epsilon_{o}}{\lambda-U_{s}-3 \epsilon}\right\} \leq \xi .
\end{aligned}
$$


Thus, $\mathcal{E}$ is a subset of the event in (5). Therefore, if (6)-(9) hold, $P\{\mathcal{E}\} \geq 0.6$, and (5) is implied. So to complete the proof, it remains to prove (6)-(9).

The process $A$ is a Poisson process with rate $\lambda$, and $Z$ is stochastically dominated by a Poisson process with rate $U_{s}$. Thus, both (6) and (7) follow from Kingman's moment bound (see Lemma VI.2 in the appendix) and the conditions in (4) on $B$.

Turning next to the proof of (8), we shall use the following observation about stochastic domination (the notion of stochastic domination is reviewed in the appendix). The observation is a mathematical version of the statement that the number of young peers remains roughly bounded because peers don't stay young for long.

Lemma III.1 The process $Y$ is stochastically dominated by the number of customers in an $M / G I / \infty$ queueing system with initial state zero, arrival rate $\lambda$, and service times having the Gamma distribution with parameters $K-1$ and $\mu / 2$.

Proof: The idea of the proof is to show how, with a possible enlargement of the underlying probability space, an $M / G I / \infty$ system can be constructed on the same probability space as the original system, so that for any time $t, Y_{t}$ is less than or equal to the number of peers in the $M / G I / \infty$ system. Let the $M / G I / \infty$ system have the same arrival process as the original systemit is a Poisson process of rate $\lambda$. For any young peer, the intensity of downloads from the one club (i.e. from any peer in the one club) is always greater than or equal to $\mu / 2$ for the original system, where we use the fact $1-\xi>1 / 2$, which is true by (1) and the assumption $K \geq 2$. We can thus suppose that each young peer has an internal Poisson clock, which generates ticks at rate $\mu / 2$, and is such that whenever the internal clock of a young peer ticks, that young peer downloads a piece from the one club. We declare that a peer remains in the $M / G I / \infty$ system until its internal clock ticks $K-1$ times. This gives the correct service time distribution, and the service times of different peers in the $M / G I / \infty$ system are independent, as required. A young peer can possibly leave the original system sooner than it leaves the $M / G I / \infty$ system, because a young peer in the original system can possibly download pieces at times when its internal clock doesn't tick. But if a young peer is still in the original system, it is in the $M / G I / \infty$ system.

Given this lemma, (8) follows from Lemma VI.4 with $m$ in the lemma equal to $2(K-1) / \mu$, and $\epsilon$ in the lemma equal to $\epsilon_{o}$, and (2). It remains to prove (9).

Consider the following construction of a stochastic system that is similar to the original one, with random variables that have similar interpretations, but with different joint distributions. We call it the comparison system. It focuses on the infected peers and the uploads by infected peers, and it is specified in Table I.

It should be clear to the reader that both the original system and the comparison system can be constructed on the same underlying probability space such that any infected peer in the original system at a given time is also in the comparison system. When such a peer becomes infected in the original system, we require that it also arrives to the comparison system, it discards all pieces it may have downloaded before becoming infected, and it subsequently ignores all opportunities to download except those occurring at the times its internal clock (described in the proof of Lemma III.1) ticks. Because infected young peers possibly stay longer in the comparison system than in the original system, some of the peers in the comparison system correspond to peers that already departed from the original system. There can also be some infected peers in the comparison system that never existed in the original system because the arrival rate of infected peers to the comparison system is greater than the arrival rate for the original system. But whenever there is an 
TABLE I

SPECIFICATION OF COMPARISON SYSTEM

\begin{tabular}{|l|l|}
\hline \multicolumn{1}{|c|}{ Original system } & \multicolumn{1}{c|}{ Comparison system } \\
\hline $\begin{array}{l}\text { The fixed seed creates infected peers at a } \\
\text { rate less than } \xi U_{s} .\end{array}$ & $\begin{array}{l}\text { The fixed seed creates infected peers at rate } \\
\xi U_{s} .\end{array}$ \\
\hline $\begin{array}{l}\text { An infected peer creates new infected peers } \\
\text { at a rate less than } \xi \mu .\end{array}$ & $\begin{array}{l}\text { An infected peer creates new infected peers } \\
\text { at rate } \xi \mu .\end{array}$ \\
\hline $\begin{array}{l}\text { An infected peer uploads piece one to one- } \\
\text { club peers at a rate less than or equal to } \\
\mu .\end{array}$ & $\begin{array}{l}\text { An infected peer uploads piece one to one- } \\
\text { club peers at rate } \mu .\end{array}$ \\
\hline $\begin{array}{l}\text { Just after a peer becomes infected, it re- } \\
\text { quires at most } K-1 \text { additional pieces, and } \\
\text { the rate for acquiring those pieces is greater } \\
\text { than or equal to } \mu / 2 .\end{array}$ & $\begin{array}{l}\text { After a new infected peer arrives, it must } \\
\text { download } K-1 \text { additional pieces, and the } \\
\text { rate for acquiring those pieces is } \mu / 2 .\end{array}$ \\
\hline
\end{tabular}

infected peer in the original system, that peer is also in the comparison system, and the following property holds. Whenever any one of the following events happens in the original system, it also happens in the comparison system:

- The fixed seed creates an infected peer.

- An infected peer creates an infected peer

- An infected peer uploads piece one to a one-club peer

Events of the second and third type just listed correspond to the two possible ways that infected peers can upload piece one. Therefore, the property implies the following lemma, where $\widehat{D}$ is the cumulative number of uploads of piece one by infected peers, up to time $t$, in the comparison system.

Lemma III.2 The process $\left(D_{t}: t \geq 0\right)$ is stochastically dominated by $\left(\widehat{D}_{t}: t \geq 0\right)$.

We can identify two kinds of infected peers in the comparison system-the root peers, which are those created by the fixed seed, and the infected peers created by other infected peers. We can imagine that each root peer affixes its unique signature on the copy of piece one that it receives from the fixed seed. The signature is inherited by all copies of piece one subsequently generated from that piece through all generations of the replication process, in which infected peers upload piece one when creating new infected peers. In this way, any upload of piece one by an infected peer can be traced back to a unique root peer. In summary, the jumps of $\widehat{D}$ can be partitioned according to which root peer generated them. Of course, the jumps of $\widehat{D}$ associated with a root peer happen after the root peer arrives. Let $\left(\widehat{\widehat{D}}_{t}: t \geq 0\right)$ denote a new process which results when all of the uploads of piece one generated by a root peer (in the comparison system) are counted at the arrival time of the root peer. Since $\widehat{\widehat{D}}$ counts the same events as $\widehat{D}$, but does so earlier, $\widehat{D}_{t} \leq \widehat{\widehat{D}}_{t}$ for all $t \geq 0$. In view of this and Lemma III.2, it is sufficient to prove (9) with $D$ replaced by $\widehat{\widehat{D}}$.

The random process $\widehat{\widehat{D}}$ is a compound Poisson process. Jumps occur at the arrival times of root peers in the comparison system, which form a Poisson process of rate $\xi U_{s}$. Let $J$ denote the size of the jump of $\widehat{\widehat{D}}$ associated with a typical root peer. The distribution of $J$ can be described by referring to an $M / G I / 1$ queueing system with arrival rate $\xi \mu$ and service times having the distribution of a random variable $\widehat{X}$ which has the Gamma distribution with parameters $K-1$ and $\mu / 2$. Note that $\rho$ in $(1)$ is the usual load factor for the reference queueing system: $\rho=\xi \mu E[\widehat{X}]$. 
The reference queueing system is similar to the number of infected peers in the comparison system, except that the customers in the $M / G I / 1$ queueing system are served one at a time. We have $J=J_{1}+J_{2}$, where

- $J_{1}$ is the number of infected peers that are descendants of the root peer (not counting the root peer itself.) That includes peers directly created by the root peer, peers created by peers created by the root peer, and so on, for all generations. $J_{1}$ has the same distribution as the number of customers in a busy period of the reference queueing system, not counting the customer that started the busy period.

- $J_{2}$ is the number of uploads of piece one to one-club peers by either the root peer or any of the descendants of the root peer. The sum of all the times that the root peer and its descendants are in the comparison system is the same as the duration, $L$, of a busy period of the reference queueing system. While in the comparison system, those peers upload piece one to the one club with intensity $\mu$. So $E\left[J_{2}\right]=\mu E[L]$ and $E\left[J_{2}^{2}\right]=\mu^{2} E[L]^{2}+\mu E[L]$.

Using this stochastic description, the formulas for the busy period in an $M / G I / 1$ queueing system ([18) and (19) in the appendix), and the facts $\rho<1 / 2, E[\widehat{X}]=2(K-1) / \mu$, and $\operatorname{Var}(\widehat{X})=$ $(K-1)(2 / \mu)^{2}$, yields

$$
\begin{aligned}
E[J] & =E\left[J_{1}\right]+E\left[J_{2}\right]=\frac{1+\mu E[\widehat{X}]}{1-\rho}-1 \\
& \leq 2[1+2(K-1)] \leq 4 K
\end{aligned}
$$

and

$$
\begin{gathered}
E\left[J_{1}^{2}\right] \leq E\left[\left(J_{1}+1\right)^{2}\right]=\frac{1+\left(\xi U_{s}\right)^{2} \operatorname{Var}(\widehat{X})}{(1-\rho)^{3}} \leq \frac{1+\rho^{2}}{(1-\rho)^{3}} \\
\begin{aligned}
E\left[J_{2}^{2}\right] & =E\left[E\left[J_{2}^{2} \mid L\right]\right]=\mu E[L]+\mu^{2} E\left[L^{2}\right] \\
& =\frac{\mu E[\widehat{X}]}{1-\rho}+\frac{\mu^{2} E\left[\widehat{X}^{2}\right]}{(1-\rho)^{3}}
\end{aligned}
\end{gathered}
$$

$$
\begin{aligned}
& E\left[J^{2}\right] \\
& \quad=E\left[\left(J_{1}+J_{2}\right)^{2}\right] \leq 2\left\{E\left[J_{1}^{2}\right]+E\left[J_{2}^{2}\right]\right\} \\
& \quad \leq 16\left\{2+\mu E[\widehat{X}]+\mu^{2} E\left[\widehat{X}^{2}\right]\right\} \\
& \quad=16\left\{2+2(K-1)+4(K-1)+4(K-1)^{2}\right\} \\
& \quad=16\left\{4 K^{2}-2 K\right\} \leq 64 K^{2}
\end{aligned}
$$

Thus, $\widehat{\widehat{D}}$ is a compound Poisson process with arrival rate of batches equal to $\xi U_{s}$ and batch sizes with first and second moments of the batch sizes bounded by $4 K$ and $64 K^{2}$ respectively. Hence, (9) with $D$ replaced by $\widehat{\widehat{D}}$ follows from Corollary VI.3 and (3). The proof of Proposition II.1) (i) is complete.

Remark III.3 We briefly explain why the comparison system was introduced in the above proof, to provide a better understanding of the proof technique. The intuitive idea behind the definition of the comparison system is that it is based on worst case assumptions regarding the number of peers that are infected by the fixed seed (i.e. the number of root peers) and the number of uploads of piece one that can be caused by each root peer. The advantage is then that the arrivals of root 
peers form a Poisson process and the total number of uploads of piece one that can be traced back to different root peers are independent in the comparison system, so that Kingman's bound for compound Poisson processes, which is a form of the law of large numbers, can be applied.

\section{Proof of Stability IF $\lambda<U_{s}$}

Proposition II.1(ii) is proved in this section, using the version of the Foster-Lyapunov stability criterion given in the appendix, and the intuition given in the last paragraph of Section II.

If $V$ is a function on the state space $\mathcal{S}$, then $Q V$ is the corresponding drift function, defined by $Q V(\mathbf{x})=\sum_{\mathbf{y}: \mathbf{y} \neq \mathbf{x}} q(\mathbf{x}, \mathbf{y})[V(\mathbf{y})-V(\mathbf{x})]$. If, as usual, the diagonal entries of $Q$ are defined to make the row sums zero, then the drift function is also given by matrix-vector multiplication: $Q V(\mathbf{x})=\sum_{\mathbf{y}} q(\mathbf{x}, \mathbf{y}) V(\mathbf{y})$.

Suppose $\lambda<U_{s}$. Given a state $\mathbf{x}$, let $n_{i}(\mathbf{x})=\sum_{c \in \mathcal{C}:|c|=i} x_{c}$. That is, $n_{i}(\mathbf{x})$ is the number of peers with precisely $i$ pieces. When the dependence on $\mathbf{x}$ is clear, we write $n_{i}$ instead of $n_{i}(\mathbf{x})$. We shall use the Foster-Lyapunov criteria with the following potential function: $V(\mathbf{x})=\sum_{i=0}^{K-1} b_{i} \Phi_{i}(\mathbf{x})$ where $b_{0}, \ldots, b_{K-1}$ are positive constants and $\Phi_{i}(\mathbf{x})=\frac{\left(n_{0}+\cdots+n_{i}\right)^{2}}{2}$.

Let $D_{i}(\mathbf{x})$ denote the sum, over all $n_{i}$ peers with $i$ pieces, of the download rates of those peers. Since any peer with $i+1$ or more pieces always has a useful piece for a peer with $i$ pieces, it follows that $D_{i}(\mathbf{x}) \geq d_{i}(\mathbf{x})$, where

$$
d_{i}(\mathbf{x})=\frac{n_{i}\left(U_{s}+\mu \sum_{j=i+1}^{K-1} n_{j}\right)}{|\mathbf{x}|} .
$$

We shall write $d_{i}$ instead of $d_{i}(\mathbf{x})$. We have

$$
\begin{aligned}
& Q \Phi_{i}(\mathbf{x}) \\
& \leq \frac{\lambda\left[\left(n_{0}+\cdots+n_{i}+1\right)^{2}-\left(n_{0}+\cdots+n_{i}\right)^{2}\right]}{2}+ \\
& \quad \frac{d_{i}\left[\left(n_{0}+\cdots+n_{i}-1\right)^{2}-\left(n_{0}+\cdots+n_{i}\right)^{2}\right]}{2} \\
& =\quad\left(\lambda-d_{i}\right)\left[n_{0}+\cdots+n_{i}\right]+\frac{\lambda+d_{i}}{2} \\
& \leq \lambda\left[n_{0}+\cdots+n_{i}+\frac{1}{2}\right]-\left(n_{i}-\frac{1}{2}\right) d_{i}
\end{aligned}
$$

Since $Q V=\sum_{i=0}^{K-1} b_{i} Q \Phi_{i}$ it follows that

$$
Q V(\mathbf{x}) \leq \frac{a_{o} \lambda}{2}+\left(\lambda \sum_{i=0}^{K-1} n_{i} a_{i}\right)-\sum_{i=0}^{K-1}\left(n_{i}-\frac{1}{2}\right) b_{i} d_{i}
$$

where $a_{i}=b_{i}+\cdots+b_{K-1}$ for $0 \leq i \leq K-1$. In what follows, assume that the constants $b_{0}, \ldots, b_{n}$ are chosen so that $1=b_{K-1}<b_{K-2}<\cdots<b_{1}<b_{0}$ and

$$
b_{i}>\left(\frac{\lambda}{U_{s}-\lambda}\right) a_{i+1} \text { for } 0 \leq i \leq K-2 .
$$

Since $a_{i+1}=a_{i}-b_{i}, 12$ is equivalent to

$$
U_{s} b_{i}-\lambda a_{i}>0 \text { for } 0 \leq i \leq K-2 .
$$


The following two lemmas and their proofs correspond to the two cases described in the intuitive description given in the last paragraph of Section III.

Lemma IV.1 There exist positive values $\eta, \epsilon$, and $L$ so that $Q V(\mathbf{x}) \leq-\epsilon|\mathbf{x}|$ whenever: $|\mathbf{x}| \geq L$ and, for some $i, n_{i} \geq(1-\eta)|\mathbf{x}|$.

Lemma IV.2 Let $\eta$ be as in Lemma IV.1. There exist positive values $\epsilon^{\prime}$ and $L^{\prime}$ so that $Q V(\mathbf{x}) \leq$ $-\epsilon^{\prime}|\mathbf{x}|$ whenever: $|\mathbf{x}| \geq L^{\prime}$ and, for all $i, n_{i} \leq(1-\eta)|\mathbf{x}|$.

Lemmas IV.1 and IV.2 imply that $Q V(\mathbf{x})<-\min \left\{\epsilon^{\prime}, \epsilon\right\}|\mathbf{x}|$ whenever $|\mathbf{x}|>\max \left\{L, L^{\prime}\right\}$, so that $Q$ and $V$ satisfy the conditions of Proposition VI.6 with $f(\mathbf{x})=\min \left\{\epsilon^{\prime}, \epsilon\right\}|\mathbf{x}|$ and $g(\mathbf{x})=$ $B \mathbb{1}_{\left\{|\mathbf{x}| \mid \leq \max \left\{L, L^{\prime}\right\}\right\}}$ where $B=\max \left\{Q V(\mathbf{x}):|\mathbf{x}| \leq \max \left\{L, L^{\prime}\right\}\right\}$. Therefore, to complete the proof of Proposition II.1(ii) it remains to prove Lemmas IV.1 and IV.2.

Proof: (Proof of Lemma IV.1.) It suffices to prove the lemma for an arbitrary choice of $i$. So fix $i \in\{0,1,2, \ldots K-1\}$, and consider a state $\mathbf{x}$ such that $n_{i} /|\mathbf{x}|>1-\eta\left(\right.$ and, in particular, $\left.n_{i} \geq 1\right)$. Then for any $j \neq i, n_{j} / n_{i}=\left(n_{j} /|\mathbf{x}|\right)\left(|\mathbf{x}| / n_{i}\right)<\frac{\eta}{1-\eta}$. Use (10) and (11) and an interchange of summation $\left(\sum_{i=0}^{K-1} \sum_{j=i+1}^{K-1}=\sum_{j=1}^{K-1} \sum_{i=0}^{j-1}\right)$ to get

$$
\begin{aligned}
& Q V(\mathbf{x}) \\
& \leq \quad \frac{a_{0} \lambda}{2}+n_{i}\left(a_{i}+\sum_{j=0, j \neq i}^{K-1} \frac{n_{j}}{n_{i}} a_{j}\right) \lambda-\left(n_{i}-\frac{1}{2}\right) b_{i} d_{i} \\
& \leq \quad \frac{a_{0} \lambda}{2}+n_{i} a_{i}\left(1+\frac{K a_{0}}{a_{i}} \frac{\eta}{1-\eta}\right) \lambda \\
& \quad-\left(n_{i}-\frac{1}{2}\right) b_{i} \frac{n_{i}\left(U_{s}+\mu \sum_{j=i+1}^{K-1} n_{j}\right)}{|\mathbf{x}|} \\
& \leq \quad \frac{a_{0} \lambda}{2} \\
& \quad+n_{i}\left\{a_{i}\left(1+\frac{K a_{0}}{a_{i}} \frac{\eta}{1-\eta}\right) \lambda-b_{i}(1-\eta) U_{s}+\frac{b_{i} U_{s}}{2|\mathbf{x}|}\right\}
\end{aligned}
$$

Notice that according to $(13)$,

$$
\begin{array}{r}
\lim _{\eta \rightarrow 0}\left\{a_{i}\left(1+\frac{K a_{0}}{a_{i}} \frac{\eta}{1-\eta}\right)\right. \\
\left.\lambda-b_{i}(1-\eta) U_{s}\right\} \\
=a_{i} \lambda-b_{i} U_{s}<0,
\end{array}
$$

and

$$
\lim _{|\mathbf{x}| \rightarrow \infty} \frac{b_{i} U_{s}}{2|\mathbf{x}|}=0 .
$$

Thus, if $\eta$ is small enough and $|\mathbf{x}|$ is large enough, the quantity within braces in (14) is negative. Therefore, if $\eta$ and $\epsilon$ are small enough, and $L$ is large enough,

$$
Q V(\mathbf{x}) \leq \frac{a_{0} \lambda+n_{i}\left\{a_{i} \lambda-b_{i} U_{s}\right\}}{2} \leq-\epsilon|\mathbf{x}|
$$

under the conditions of the lemma, whenever $|\mathbf{x}| \geq L$. Lemma IV.1 is proved. 
Proof: (Proof of Lemma IV.2, Let $\eta$ be given by Lemma IV.1, and consider a state $\mathbf{x}$ such that $n_{i} /|\mathbf{x}| \leq 1-\eta$ for all $i$. It follows that there exists $i_{1}$ and $i_{2}$ with $0 \leq i_{1}<i_{2} \leq K-1$ such that $n_{i_{1}} \geq \frac{\eta|\mathbf{x}|}{K}$ and $n_{i_{2}} \geq \frac{\eta|\mathbf{x}|}{K}$. Then

$$
\begin{aligned}
& Q V(\mathbf{x}) \\
& \leq \frac{a_{0} \lambda}{2}+|\mathbf{x}| a_{0} K \lambda-\left(n_{i_{1}}-\frac{1}{2}\right) b_{i_{1}} d_{i_{1}} \\
&=\frac{a_{0} \lambda}{2}+|\mathbf{x}| a_{0} K \lambda \\
&-\left(n_{i_{1}}-\frac{1}{2}\right) b_{i_{1}} \frac{n_{i_{1}}\left(U_{s}+\mu \sum_{j=i_{1}+1}^{K-1} n_{j}\right)}{|\mathbf{x}|} \\
& \leq \frac{a_{0} \lambda}{2}+|\mathbf{x}| a_{0} K \lambda-\left(\frac{\eta|\mathbf{x}|}{K}-\frac{1}{2}\right) b_{i_{1}} \frac{\eta^{2}|\mathbf{x}|}{K^{2}} \mu \\
& \leq \frac{a_{0} \lambda}{2}+|\mathbf{x}|\left\{a_{0} K \lambda+\frac{b_{0} \mu}{2}\right\}-\left(\frac{\eta}{K}\right)^{3}|\mathbf{x}|^{2} \mu
\end{aligned}
$$

The conclusion of the lemma follows because of the term in 15$]$ that is quadratic in $|\mathbf{x}|$.

\section{Generalization AND Discussion}

\section{A. General Piece Selection Policies}

A piece selection policy is used by a peer to choose which piece to download whenever it contacts another peer. The random useful piece selection policy is assumed above, but the results extend to a large class of piece selection policies. Essentially the only restriction needed is that if the contacted peer has a useful piece for the contacting peer, then a useful piece must be downloaded. This restriction is similar to a work conserving restriction in the theory of service systems. In particular, the results hold for a broad class of rarest first piece selection policies. Peers can estimate which pieces are more rare in a distributed way, by exchanging information with the peers they contact. Even more general policies would allow the piece selection to depend in an arbitrary way on the piece collections of all peers. Interestingly enough, the results extend even to seemingly bad piece selection policies. For example, it includes the sequential piece selection policy, in which peers obtain the pieces in order, beginning with piece one. The sequential policy can be viewed as a most abundant first useful piece selection policy, or just the opposite of rarest piece first.

To be specific, consider the following family $\mathcal{H}$ of piece selection policies. Each policy in $\mathcal{H}$ corresponds to a mapping $h$ from $\mathcal{C} \times(\mathcal{C} \cup\{\mathcal{F}\}) \times \mathcal{S}$ to the set of probability distributions on $\mathcal{F}$, satisfying the usefulness constraint:

$$
\sum_{i \in B-A} h_{i}(A, B, \mathbf{x})=1 \text { whenever } B \not \subset A
$$

with the following meaning of $h$ :

- When a type $A$ peer selects a piece to download from a type $B$ peer and the state of the entire network is $\mathbf{x}$, piece $i$ is selected with probability $h_{i}(A, B, \mathbf{x})$, for $i \in \mathcal{F}$.

- When the fixed seed selects a piece to upload to a type $A$ peer and the state of the entire network is $\mathbf{x}$, piece $i$ is selected with probability $h_{i}(A, \mathcal{F}, \mathbf{x})$, for $i \in \mathcal{F}$.

The piece selection policies noted above are included in $\mathcal{H}$. 
Reconsider the proof of transience in Section III under a piece selection policy in $\mathcal{H}$. From any state it is possible to reach the empty state, and from the empty state it is possible to reach a state with one peer in the network having all pieces except some piece $i_{0}$. From that state, for any $N_{o} \geq 1$, it is possible to reach the state with $N_{o}$ peers missing only piece $i_{0}$, and no other peers in the network. It may be impossible for $i_{0}$ to equal one, but by renumbering the pieces if necessary, it can be assumed without loss of generality that $i_{0}$ is one. Thus, whatever piece selection policy in $\mathcal{H}$ is applied, beginning from any initial state, for any $N_{o} \geq 1$, in a finite time with a positive probability, the system can arrive into the state where there are $N_{o}$ peers and all of them are one-club peers. Thus, as in Section III to prove transience it suffices to show that from such an initial state, there is a positive probability that the number of peers converges to infinity. The arrival rate of new peers and the upload rate of the seed does not depend on the piece selection policy, so (6) and (7) are valid for any piece selection policies in $\mathcal{H}$. Moreover, Lemma III.1 and Lemma III.2 are valid for any piece selection policies in $\mathcal{H}$ because the two lemmas depend on the properties that peer selection is uniformly random and the piece selection is useful if a useful piece is available. Therefore (8) and (9) are also valid for any piece selection policy in $\mathcal{H}$. Thus, we conclude that the proof of Proposition II.1(i) in Section III works for any piece selection policy in $\mathcal{H}$.

Reconsider next the proof of positive recurrence in Section IV, but for an arbitrary piece selection policy in $\mathcal{H}$. The inequalities developed for the proofs of Lemmas IV.1 and IV.2 hold with the same Lyapunov function; useful piece selection suffices. Thus, if $\lambda<U_{s}$, it can be shown that the Lyapunov stability condition, namely $Q V(\mathbf{x}) \leq-\epsilon|\mathbf{x}|$, for $|\mathbf{x}|$ sufficiently large, still holds. The final conclusion has to be modified, however, because under some policies in $\mathcal{H}$, the Markov process might no longer be irreducible. For example, with the sequential useful piece selection policy, the set of states such that every peer holds a set of pieces of the form $\{1,2, \ldots, J\}$ for some $J$ with $0 \leq J \leq K-1$, is a closed subset of states, in the terminology of classification of states of discrete-state Markov processes. In general, the set of all states that are reachable from the empty state is the unique minimal closed set of states, and the process restricted to that set of states is irreducible. By a minor variation of the Foster-Lyapunov stability proposition, the Lyapunov stability condition implies that the Markov process restricted to that closed set of states is positive recurrent, and the mean time to reach the empty state beginning from an arbitrary initial state is finite.

We summarize the discussion of the previous two paragraphs as a proposition.

Proposition V.1 (Stability conditions for general useful piece selection policies) Suppose a useful piece selection policy from $\mathcal{H}$ is used, for a network with random peer contacts and parameters $K, \lambda, U_{s}$, and $\mu$ as in Section II] There is a single class of closed states containing the empty state, and all other states are transient. (i) If $\lambda>U_{s}$ then the Markov process is transient, and the number of peers in the system converges to infinity with probability one. (ii) If $\lambda<U_{s}$ the Markov process with generator $Q$ restricted to the closed set of states is positive recurrent, the mean time to reach the empty state from any initial state has finite mean, and the equilibrium distribution $\pi$ is such that $\sum_{\mathbf{x}} \pi(\mathbf{x})|\mathbf{x}|<\infty$.

Thus, with the exception of the borderline case $\lambda=\mu$, rarest first piece selection does not increase the region of stability, nor does most abundant first piece selection decrease the region of stability.

\section{B. Network Coding}

Network coding, introduced by Ahlswede, Cai, and Yeung, [1], can be naturally incorporated into P2P distribution networks, as noted in [4]. The related work [3] considers all to all exchange 
of pieces among a fixed population of peers through random contacts and network coding. The method can be described as follows. The file to be transmitted is divided into $K$ data pieces, $m_{1}, m_{2}, \ldots, m_{K}$. The data pieces are taken to be vectors of some fixed length $r$ over a finite field $\mathbb{F}_{q}$ with $q$ elements, where $q$ is some power of a prime number. If the piece size is $M$ bits, this can be done by viewing each message as an $r=\left\lceil M / \log _{2}(q)\right\rceil$ dimensional vector over $\mathbb{F}_{q}$. Any coded piece $e$ is a linear combination of the original $K$ data pieces: $e=\sum_{i=1}^{K} \theta_{i} m_{i}$; the vector of coefficients $\left(\theta_{1}, \ldots, \theta_{K}\right)$ is called the coding vector of the coded piece; the coding vector is included whenever a coded piece is sent. The fixed seed uploads coded pieces to peers, and peers exchange coded pieces. In this context, the type of a peer $A$ is the subspace $V_{A}$ of $\mathbb{F}_{q}^{K}$ spanned by the coding vectors of the coded pieces it has received. Once the dimension of $V_{A}$ reaches $K$, peer $A$ can recover the original message.

When peer A contacts peer B, suppose peer B sends peer A a random linear combination of its coded pieces, where the coefficients are independent and uniformly distributed over $\mathbb{F}_{q}$. Equivalently, the coding vector of the coded piece sent from $B$ is uniformly distributed over $V_{B}$. The coded piece is considered useful to $A$ if adding it to $A$ 's collection of coded pieces increases the dimension of $V_{A}$. Equivalently, the piece from $B$ is useful to $A$ if its coding vector is not in the subspace $V_{A} \cap V_{B}$. The probability the piece is useful to $A$ is therefore given by

$$
P\{\text { piece is useful }\}=\frac{\left|V_{A} \cap V_{B}\right|}{\left|V_{B}\right|}=1-q^{\operatorname{dim}\left(V_{A} \cap V_{B}\right)-\operatorname{dim}\left(V_{B}\right)} .
$$

If peer $B$ can possibly help peer $A$, meaning $V_{B} \not \subset V_{A}$ (true, for example, if $\operatorname{dim}\left(V_{B}\right)>\operatorname{dim}\left(V_{A}\right)$ ), the probability that a random coded piece from $\mathrm{B}$ is helpful to $\mathrm{A}$ is greater than or equal to $1-\frac{1}{q}$. The probability a random coded piece from the seed is useful to a peer $A$ with $\operatorname{dim}\left(V_{A}\right)=K-1$ is precisely $1-\frac{1}{q}$. Therefore, when all peers have the same state and the common state has dimension $K-1$, the departure rate from the network is $\widetilde{U}_{s}=U_{s}\left(1-\frac{1}{q}\right)$.

The network state $\mathrm{x}$ specifies the number of peers in the network of each type. There are only finitely many types, so the overall state space is still countably infinite. Moreover, the Markov process is easily seen to be irreducible.

Reconsider the proof of transience in Section III, but now under network coding. Fix any subspace $V^{-}$of $\mathbb{F}_{q}^{K}$ with dimension $K-1$. Call a peer a one-club peer if its state is $V^{-}$. For any $N_{o} \geq 1$, it is possible to reach the state with $N_{o}$ one-club peers and no other peers in the network. As before, call a peer a young peer if it is not a one-club peer. In the case of network coding, call a peer infected if its state is not a subspace of $V^{-}$. The only way a peer can become infected is by downloading a piece either from the seed or from an infected peer. Lemmas 6 and 7 are valid for network coding, if the condition $\lambda>U_{s}$ is replaced by $\lambda>U_{s}$. Moreover, Lemma III.1 and Lemma III.2 are valid for network coding because the two lemmas depend on the properties that peer selection is uniformly random and the rate useful pieces are delivered by the seed to one-club peers is arbitrarily close to $\widetilde{U}_{s}$. Thus, we conclude that Proposition II.1.i) in Section III, with $U_{s}$ replaced by $\widetilde{U}_{s}$, extends to the case of network coding.

Reconsider the proof of positive recurrence in Section IV, but with random useful piece selection replaced by network coding as described, and $U_{s}$ replaced by $\widetilde{U}_{s}=U_{s}\left(1-\frac{1}{q}\right)$. Suppose the same Lyapunov function is used, except the new meaning of $n_{i}(\mathbf{x})$, or $n_{i}$ for short, is the number of peers $A$ with $\operatorname{dim}\left(V_{A}\right)=i$. Lemmas IV.1 and IV.2 are valid for network coding, if the condition $\lambda<U_{s}$ is replaced by $\lambda<\widetilde{U}_{s}$. Thus, if $\lambda<U_{s}$, it can be shown that the Lyapunov stability condition, namely $Q V(\mathbf{x}) \leq-\epsilon|\mathbf{x}|$, for $|\mathbf{x}|$ sufficiently large, still holds, and the Foster-Lyapunov stability criterion applies. 
We summarize the discussion of the previous two paragraphs as a proposition.

Proposition V.2 (Stability conditions for network coding based system) Suppose random linear network coding with vectors over $\mathbb{F}_{q}^{K}$ is used, with random peer contacts and parameters $K, \lambda, U_{s}$, and $\mu$ as in Section II. (i) If $\lambda>U_{s}\left(1-\frac{1}{q}\right)$ then the Markov process is transient, and the number of peers in the system converges to infinity with probability one. (ii) If $\lambda<U_{s}\left(1-\frac{1}{q}\right)$ the Markov process is positive recurrent, and the equilibrium distribution $\pi$ is such that $\sum_{\mathbf{x}} \pi(\mathbf{x})|\mathbf{x}|<\infty$.

Thus, as $q \rightarrow \infty$, the stability region for the system with network coding converges to that for useful piece selection. Network coding has the advantage that no exchange of state information among peers is needed because there is no need to identify useful pieces.

\section{Peer Seeds}

In many unstructured peer-to-peer systems, such as BitTorrent, peers often dwell in the network awhile after they have collected all the pieces. In effect, these peers temporarily become seeds, called peer seeds. The uploading provided by peer seeds is able to mitigate the missing piece syndrome and enlarge the stability region. Intuitively, if every peer can upload, on average, just one more piece after collecting all pieces, then every peer can help one one-club peer to depart, so the missing piece syndrome would not persist. This is explored for the case of $K=1$ and $K=2$ (for the sequential piece selection policy) in [8] and for random useful piece selection with arbitrary $K \geq 1$ in [17].

\section{Peer Selection and Tit-for-Tat}

Another way to overcome the missing piece syndrome relies on peer selection policies. For instance, if young peers contact infected peers preferentially, or if the seed uploads to young peers preferentially, the network can be stabilized by the resulting increase in the number of infected peers. So some sort of coordination policy, providing the identification of rare pieces and young peers, and the transmission of the rare pieces to the young peers, can counter the missing piece syndrome. A mechanism built into BitTorrent, called tit-for-tat operation, may alter the peer selection policy enough to yield stability for any choice of $\lambda, \mu$, and $U_{s}$. Under tit-for-tat operation, peers upload almost exclusively to peers from which they can simultaneously download. An obvious benefit of tit-for-tat is to give peers incentive to upload, thereby helping other peers, but it also may be effective against the missing piece syndrome. Specifically, tit-for-tat encourages one-club peers to reduce their rate of download to the young peers, because the young peers have nothing to upload to the one-club members. This increases the amount of time that peers remain young, giving them a greater chance to obtain a rare piece from the fixed seed. Also, infected peers would preferentially send to young peers, because often a normal young peer and an infected young peer would be able to help each other. While it is thus clear that tit-for-tat operation helps combat the missing piece syndrome, we leave open the problem of quantifying the effect for a specific model.

\section{E. The Borderline of Stability}

We have shown that, for any $\mu>0$, the system is stable if $\lambda<U_{s}$ and unstable if $\lambda>U_{s}$, and this result is insensitive to the value of $\mu$ and to the piece selection policy, as long as a useful piece is selected whenvever possible. While it may not be interesting from a practical point of 


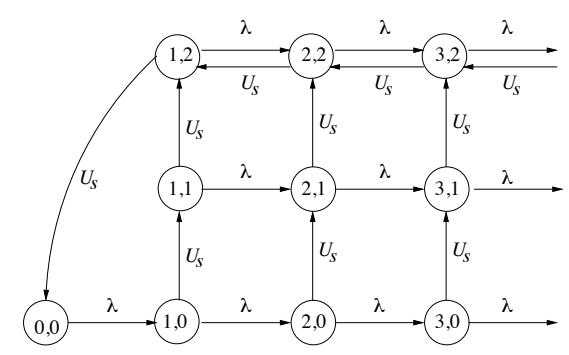

Fig. 4. Transition rates for $\mu=\infty$ system for $K=3$.

view, we comment on the case $\lambda=U_{s}$. First, we give a precise result for a limiting case of the original system, and then we offer a conjecture.

A simpler network model results by taking a limit as $\mu \rightarrow \infty$. Call a state slow if all peers in the system have the same type, which includes the state such that there are no peers in the system. Otherwise, call a state fast. The total rate of transition out of any slow state does not depend on $\mu$, and the total rate out of any fast state is greater than or equal to $\mu / 2$. For very large values of $\mu$, the process spends most of its time in slow states. The original Markov process can be transformed into a new one by watching the original process while it is in the set of slow states. This means removing the portions of each sample path during which the process is in fast states, and timeshifting the remaining parts of the sample path to leave no gaps in time. The limiting Markov process, which we call the $\mu=\infty$ process, is the weak limit (defined as usual for probability measures on the space of càdlàg sample paths equipped with the Skorohod topology) of the original process watched in the set of slow states, as $\mu \rightarrow \infty$. By symmetry of the model, the state space of the $\mu=\infty$ process can be reduced further, to $\widehat{\mathcal{S}}=\{(0,0)\} \cup\{(n, k): n \geq 1,1 \leq k \leq K-1\}$, where a state $(n, k)$ corresponds to $n$ peers in the system which all possess the same set of $k$ pieces. The positive transition rates of the $\mu=\infty$ process are given by:

$$
\begin{array}{rcc}
\text { transition } & \text { rate } & \text { condition } \\
(n, k) \rightarrow(n+1, k) & \lambda & (n, k) \in \widehat{\mathcal{S}} \\
(n, k) \rightarrow(n, k+1) & U_{s} & n \geq 1,0 \leq k \leq K-2 \\
(n, K-1) \rightarrow(n-1, K-1) & U_{s} & n \geq 2, k=K-1 \\
(1, K-1) \rightarrow(0,0) & U_{s} &
\end{array}
$$

and the transition rate diagram is pictured in Figure 4 for $K=3$. The top layer of states consists of those for which all peers have $K-1$ pieces. These states correspond to all peers being in the one club, or all missing some other piece. From any state the process reaches the top layer in mean time less than or equal to $\frac{1}{\lambda}+\frac{K-1}{U_{s}}$, and within the top layer the process behaves like a birth-death process with birth rate $\lambda$ and death rate $U_{s}$. Since such birth-death processes are null-recurrent if $\lambda=U_{s}$, it follows that the $\mu=\infty$ model is null-recurrent if $\lambda=U_{s}$.

Consider the original process with $\lambda=U_{s}$ and finite $\mu$. Suppose the process is in a state with a very large one club which includes all or nearly all the peers; let $n$ be the number of peers in the one club. New young peers arrive at rate $\lambda$, and they are in the system for approximately $\frac{1}{\mu}$ time units while they are holding exactly $k$ pieces for $0 \leq k \leq K-2$. . Thus, over the short term, the mean number of young peers in the system holding $k$ pieces is near $\frac{\lambda}{\mu}$ for $0 \leq k \leq K-2$. The average fraction of peers that are young peers holding $k$ pieces is thus approximately $\frac{\lambda}{n \mu}$ for $0 \leq k \leq K-2$. The average total rate that young peers holding $k$ pieces become infected is dominated by the rate the fixed seed downloads piece one to them and is thus approximately 
$\frac{U_{s} \lambda}{(K-k) n \mu}$, where the factor $\frac{1}{K-k}$ comes the assumption of uniform random useful piece selection for downloads from the seed. A young peer that becomes infected when it has $k$ pieces will eventually release, on average, about $K-k-1$ other peers from the one club. Thus, to a first order approximation, for large $n$, the number of peers in the system behaves like a birth-death process with arrival rate $\lambda$ and state dependent departure rate $U_{s}\left(1+\frac{\mu_{o}}{n \mu}\right)$, where

$$
\mu_{o}=\lambda \sum_{k=0}^{K-2} \frac{K-k-1}{K-k} .
$$

The elementary theory of birth-death processes shows that a birth-death process with constant birth rate $\lambda$ and state-dependent death rate $\lambda\left(1+\frac{c}{n}\right)$ is positive recurrent if $c>1$ and null-recurrent if $0<c \leq 1$. This strongly suggests the following to be true:

Conjecture V.1 If $\lambda=U_{s}$, the process is positive recurrent if $0<\mu<\mu_{o}$ and is null recurrent if $\mu>\mu_{o}$.

We also expect similar results to be true for other piece selection policies, but the value of $\mu_{o}$ would depend on the piece selection policy.

\section{APPENDIX}

\section{A. Stochastic comparison}

A continuous-time random process is said to be càdlàg if, with the possible exception of a set of probability zero, the sample paths of the process are right continuous and have finite left limits.

Definition VI.1 Suppose $A=\left(A_{t}: t \geq 0\right)$ and $B=\left(B_{t}: t \geq 0\right)$ are two random processes, either both discrete-time random processes, or both continuous time, càdlàg random processes. Then $A$ is stochastically dominated by $B$ if there is a single probability space $(\Omega, \mathcal{F}, P)$, and two random processes $\tilde{A}$ and $\tilde{B}$ on $(\Omega, \mathcal{F}, P)$, such that

(a) $A$ and $\tilde{A}$ have the same finite dimensional distributions,

(b) $B$ and $\tilde{B}$ have the same finite dimensional distributions, and

(c) $P\left\{\tilde{A}_{t} \leq \tilde{B}_{t}\right.$ for all $\left.t\right\}=1$.

Clearly if $A$ is stochastically dominated by $B$, then for any $a$ and $t, P\left\{A_{t} \geq a\right\} \leq P\left\{B_{t} \geq a\right\}$.

\section{B. Appendix: Kingman's Moment bound for SII processes}

Let $\left(X_{t}: t \geq 0\right)$ be a random process with stationary, independent increments with $X_{0}=0$. Suppose the sample paths are càdlàg (i.e. right-continuous with finite left limits). Suppose $E\left[X_{1}^{2}\right]$ is finite, so there are finite constants $\mu$ and $\sigma^{2}$ such that $E\left[X_{t}\right]=\mu t$ and $\operatorname{Var}\left(X_{t}\right)=\sigma^{2} t$ for all $t \geq 0$. Let $X^{*}=\sup _{t \geq 0} X_{t}$.

Lemma VI.2 (Kingman's moment bound [6] extended to continuous time) Suppose that $\mu<0$. Then $E\left[X^{*}\right] \leq \frac{\sigma^{2}}{-2 \mu}$. Also, for any $B>0, P\left\{X^{*} \geq B\right\} \leq \frac{\sigma^{2}}{-2 \mu B}$.

Proof: For each integer $n \geq 0$, let $S^{n}$ denote the random walk process $S_{k}^{n}=X_{k 2^{-n}}$. Let $S^{n *}=\sup _{k \geq 0} S_{k}$. By Kingman's moment bound for discrete time processes,

$$
E\left[S^{n *}\right] \leq \frac{\operatorname{Var}\left(S_{1}^{n}\right)}{-2 E\left[S_{1}^{n}\right]}=\frac{\sigma^{2}}{-2 \mu}
$$


Since $S^{n *}$ is nondecreasing in $n$ and converges a.s. to $X^{*}$, the first conclusion of the lemma follows. The second conclusion follows from the first by Markov's inequality.

Corollary VI.3 Let $C$ be a compound Poisson process with $C_{0}=0$, with jump times given by a Poisson process of rate $\alpha$, and jump sizes having mean $m_{1}$ and mean square value $m_{2}$. Then for all $B>0$ and $\epsilon>\alpha m_{1}$

$$
P\left\{C_{t}<B+\epsilon t \text { for all } t\right\} \geq 1-\frac{\alpha m_{2}}{2 B\left(\epsilon-\alpha m_{1}\right)}
$$

Proof: Let $X_{t}=C_{t}-\epsilon t$. Then $X$ satisfies the hypotheses of Lemma VI.2 with $\mu=\alpha m_{1}-\epsilon$ and $\sigma^{2}=\alpha m_{2}$. So $P\left\{X^{*} \geq B\right\} \leq \frac{\alpha m_{2}}{-2\left(\alpha m_{1}-\epsilon\right) B}$, which implies (16).

\section{A maximal bound for an $\mathrm{M} / G I / \infty$ queue}

Lemma VI.4 Let $M$ denote the number of customers in an $M / G I / \infty$ queueing system, with arrival rate $\lambda$ and mean service time $m$. Suppose that $M_{0}=0$. Then for $B, \epsilon>0$,

$$
P\left\{M_{t} \geq B+\epsilon t \text { for some } t \geq 0\right\} \leq \frac{e^{\lambda(m+1)} 2^{-B}}{1-2^{-\epsilon}}
$$

Proof: Our idea is to find another $M / G I / \infty$ system whose number of customers sampled at integer times can be used to bound $M$. Suppose we let every customer for the original process stay in the system for one extra unit time after they have been served. Let $M_{t}^{\sharp}$ be the number of customers in this new $M / G I / \infty$ system at time $t$. Note that $M^{\sharp}$ is also the number in an $M / G I / \infty$ system, with arrival rate $\lambda$ and mean service time $m+1$. By a well-known property of $M / G I / \infty$ systems, for any time $t, M_{t}^{\sharp}$ is a Poisson random variable. Since the initial state is zero, the mean number in the system at any time $t$ is less than $\lambda(m+1)$, which is the mean number in the system in equilibrium. If $\operatorname{Poi}(\mu)$ represents a Poisson random variable with mean $\mu$, then the Chernoff inequality yields $P\{\operatorname{Poi}(\mu) \geq a\} \leq \exp \left(\mu\left(e^{\theta}-1\right)-\theta a\right)$, and taking $\theta=\ln 2$ yields $P\{\operatorname{Poi}(\mu) \geq a\} \leq e^{\mu} 2^{-a}$. For any integer $i \geq 1$, if $t \in(i-1]$, then $M_{t} \leq M^{\sharp}(i)$. Therefore,

$$
\begin{aligned}
P & \left\{M_{t} \geq B+\epsilon t \text { for some } t \geq 0\right\} \\
& \leq \sum_{i=1}^{\infty} P\left\{M_{t} \geq B+\epsilon t \text { for some } t \in(i-1, i]\right\} \\
& \leq \sum_{i=1}^{\infty} P\left\{M_{i}^{\sharp} \geq B+\epsilon(i-1)\right\} \\
& \leq \sum_{i=1}^{\infty} e^{\lambda(m+1)} 2^{-(B+\epsilon(i-1))} \\
& =\frac{e^{\lambda(m+1)} 2^{-B}}{1-2^{-\epsilon}}
\end{aligned}
$$


Consider an $M / G I / 1$ queue with arrival rate $\lambda$. Let $N$ denote the number of customers served in a busy period, let $L$ denote the length of a busy period, and let $X$ denote the service time of a typical customer.

Lemma VI.5 Let $\rho=\lambda E[X]$. It $\rho<1$ then

$$
\begin{gathered}
E[N]=\frac{1}{1-\rho} \quad E\left[N^{2}\right]=\frac{1+\lambda^{2} \operatorname{Var}(X)}{(1-\rho)^{3}} \\
E[L]=\frac{E[X]}{1-\rho} \quad E\left[L^{2}\right]=\frac{E\left[X^{2}\right]}{(1-\rho)^{3}} \\
\operatorname{Cov}(N, L)=\frac{\lambda E\left[X^{2}\right]}{(1-\rho)^{3}}
\end{gathered}
$$

The lemma can be proved by the well-known branching process method. Let $X$ denote the service time of a customer starting a new busy period. Let $Y$ denote the number of arrivals while the first customer is being served. Then, given $X=x$, the conditional distribution of $Y$ is Poisson with mean $\lambda x$. View any customer in the busy period that arrives after the first customer, to be the offspring of the customer in the server at the time of arrival. This gives the well known representation for $N$ and $L$ :

$$
\begin{aligned}
N & =1+\sum_{i=1}^{Y} N_{i} \\
L & =X+\sum_{i=1}^{Y} L_{i}
\end{aligned}
$$

where $\left(N_{i}, L_{i}\right), i \geq 1$ is a sequence of independent random 2-vectors such that for each $i,\left(N_{i}, L_{i}\right)$ has the same distribution as $(N, L)$. Using Wald's identity, these equations can be used to prove the lemma.

\section{E. Foster-Lyapunov stability criterion}

Proposition VI.6 Combined Foster-Lyapunov stability criterion and moment bound-continuous time (See [5], [12].) Suppose $X$ is a continuous-time, irreducible Markov process on a countable state space $\mathcal{S}$ with generator matrix $Q$. Suppose $V, f$, and $g$ are nonnegative functions on $\mathcal{S}$ such that $Q V(\mathbf{x}) \leq-f(\mathbf{x})+g(\mathbf{x})$ for all $\mathbf{x} \in \mathcal{S}$, and, for some $\delta>0$, the set $C$ defined by $C=\{\mathbf{x}: f(\mathbf{x})<g(\mathbf{x})+\delta\}$ is finite. Suppose also that $\{\mathbf{x}: V(\mathbf{x}) \leq K\}$ is finite for all $K$. Then $X$ is positive recurrent and, if $\pi$ denotes the equilibrium distribution, $\sum_{\mathbf{x}} f(\mathbf{x}) \pi(\mathbf{x}) \leq \sum_{\mathbf{x}} g(\mathbf{x}) \pi(\mathbf{x})$.

\section{REFERENCES}

[1] Ahlswede, R., CAI, N., LI, S.-Y., AND Yeung, R. (2000). Network information flow. IEEE Transactions on Information Theory 46, 4 (jul), $1204-1216$.

[2] CoHEN, B. (2003). Incentives build robustness in BitTorrent. P2PECON Workshop.

[3] Deb, S., MÉEdard, M., ANd Choute, C. (2006). Algebraic gossip: A network coding approach to optimal multiple rumor mongering. IEEE Transactions on Information Theory 52, 6 (June), 2486-2502.

[4] Gkantsidis, C. And RodrigueZ, P. (2005). Network coding for large scale content distribution. In Proceedings INFOCOM 2005. Vol. 4. 2235 - 2245 vol. 4.

[5] HAJEK, B. Notes for ECE 567: Communication network analysis. Available at www.illinois.edu/ b-hajek.

[6] Kingman, J. (1962). Some inequalities for the queue GI/G/1. Biometrika 49, 3/4, 315-324. 
[7] KURTZ, T. G. (1981). Approximation of population processes. CBMS-NSF Regional Conference Series in Applied Mathematics, Vol. 36. Society for Industrial and Applied Mathematics (SIAM), Philadelphia, Pa.

[8] Leskelä, L., Robert, P., And Simatos, F. (2010). Interacting branching processes and linear file-sharing networks. Advances in Applied Probability 42, 3, 834-854.

[9] Massoulié, L. ANd Vojnović, M. (2005). Coupon replication systems. In SIGMETRICS '05: Proceedings of the 2005 ACM SIGMETRICS international conference on Measurement and modeling of computer systems. ACM, New York, NY, USA, $2-13$.

[10] Massoulié, L. And Vojnović, M. (2008). Coupon replication systems. IEEE/ACM Trans. Networking 16, 3, $603-616$.

[11] Menasché, D. S., de Aragẽo Rocha, A. A., de Souza e Silva, E., Leão, R. M. M., Towsley, D. F., and VENKATARAMANI, A. (2010). Estimating self-sustainability in peer-to-peer swarming systems. http://arxiv.org/abs/1004.0395

[12] Meyn, S. And Tweedie, R. (2009). Markov Chains and Stochastic Stability (Cambridge Mathematical Library), 2 ed. Cambridge University Press.

[13] Norros, I., Reittu, H., And Eirola, T. (2009). On the stability of two-chunk file-sharing systems. Available on arXiv:0910.5577

[14] QIU, D. AND SRIKANT, R. (2004). Modeling and performance analysis of BitTorrent-like peer-to-peer networks. In SIGCOMM '04. ACM, New York, NY, USA, 367-378.

[15] YANG, X. AND DE VECIANA, G. (2004). Service capacity of peer to peer networks. In IEEE INFOCOM. 1-11.

[16] YANG, X. AND DE Veciana, G. (2006). Performance of peer-to-peer networks: Service capacity and role of resource sharing policies. Performance Evaluation 63, 3, 175-194.

[17] ZhU, J. AND HAJEK, B. (2011). Stability of a peer-to-peer communication system. In Proceedings SIGACT-SIGOPS Symposium on Principles of Distributed Computing. 